\title{
On the Task of Finding One Highly Relevant Document with High Precision
}

\author{
TETSUYA SAKAI ${ }^{\dagger}$
}

\begin{abstract}
This paper examines the problem of evaluating systems that aim at finding one highly relevant document with high precision. Such a task is important for modern search environments such as the Web where recall is unimportant and/or unmeasurable. Reciprocal Rank is a metric designed for finding one relevant document with high precision, but it can only handle binary relevance. We therefore introduce a new metric called $O$-measure, for high precision, high relevance search, and show (a) How the task of finding one relevant document is different from that of finding as many relevant documents as possible, in both binary and graded relevance settings; and (b) How the task of finding one highly relevant document is different from that of finding any one relevant document. We use four test collections and the corresponding sets of formal runs from the NTCIR-3 Crosslingual Information Retrieval track to compare the tasks and metrics in terms of resemblance, stability and discrimination power in system ranking.
\end{abstract}

\section{Introduction}

Traditional Information Retrieval (IR) evaluation relies on precision and recall. For example, the primary metric used for the "ad hoc" and cross-language IR tasks at TREC ${ }^{\text {in } 1}$, $\mathrm{CLEF}^{\star 2} 2$ and $\mathrm{NTCIR}^{\star 3}$ is Average Precision (AveP), designed for retrieving all relevant documents near the top of the ranked list (i.e., with both high recall and high precision). However, in modern large-scale search environments such as the $\mathrm{Web}^{5), 17)}$, recall is often unimportant or unmeasurable, and high precision search is required. In particular, finding one relevant document with high precision is a practical task: Even if there in fact are more than one relevant document, the user often stops going through a ranked document list after having read one relevant document, either knowing or assuming that all of the other relevant documents lack novelty, or additional information that may be useful to him. For evaluating retrieval of one relevant document with high precision, Reciprocal Rank (RR) has been used ${ }^{16), 17)}$.

However, high precision is merely a necessary condition for practical search environments such as the Web. What such environments also require is retrieval of highly relevant documents ${ }^{18)}$, or high relevance search. Neither AveP nor RR can handle this problem as they are based on binary relevance. We therefore introduce a new metric called $O$-measure for

† Toshiba Corporate R\&D Center finding one highly relevant document with high precision. O-measure is a variant of $Q$-measure and $R$-measure which are highly correlated with AveP but can handle graded relevance ${ }^{8), 10), 13)}$.

Figure 1 defines four IR tasks based on the discussion provided above, each with an example IR evaluation metric. Using AveP, Qmeasure, RR and O-measure, this paper shows (a) How the task of finding one relevant document is different from that of finding as many relevant documents as possible, in both binary and graded relevance settings (i.e., How Tasks $\mathrm{C}$ and $\mathrm{D}$ are different from Tasks $\mathrm{A}$ and $\mathrm{B}$ ); and (b) How the task of finding one highly relevant document is different from that of finding any one relevant document (i.e., How Task D is different from Task C). To our knowledge, the present work is the first extensive study of Task D. We have already discussed how Task B is different from Task A in Refs. 8), 10), 13).

We use four test collections and the corresponding sets of formal runs from the NTCIR-3 Crosslingual Information Retrieval (CLIR) track ${ }^{2)}$ to compare the tasks and metrics in terms of resemblance, stability and discrimination power in system ranking.

The remainder of this paper 4 is organised

婉 Text REtrieval Conference: http://trec.nist.gov/

is 2 Cross-Language Evaluation Forum: http://www.clef-campaign.org/

«3 NACSIS/NII Test Collections for Information Retrieval Systems: http://research.nii.ac.jp/ntcir/

is 4 An early, four-page version of this paper appeared in FIT 2005 Information Technology Letters ${ }^{12)}$. 


\begin{tabular}{l|l|l|}
\multicolumn{1}{c}{} & \multicolumn{1}{c}{$\begin{array}{c}\text { Binary } \\
\text { relevance }\end{array}$} & \multicolumn{1}{c}{$\begin{array}{l}\text { Graded } \\
\text { relevance }\end{array}$} \\
\cline { 2 - 3 } $\begin{array}{l}\text { Finding } \\
\text { as many } \\
\text { relevant } \\
\text { documents } \\
\text { as possible }\end{array}$ & $\begin{array}{l}\text { Task A } \\
\text { (Example metric: } \\
\text { AveP) }\end{array}$ & $\begin{array}{l}\text { Task B } \\
\text { (Example metric: } \\
\text { Q-measure) }\end{array}$ \\
\cline { 2 - 3 } $\begin{array}{l}\text { Finding ONE } \\
\text { relevant } \\
\text { document } \\
\text { only }\end{array}$ & $\begin{array}{l}\text { Task C } \\
\text { (Example metric: } \\
\text { RR) }\end{array}$ & $\begin{array}{l}\text { Task D } \\
\text { (Example metric: } \\
\text { O-measure) }\end{array}$ \\
\cline { 2 - 3 } & &
\end{tabular}

Fig. 1 Four IR tasks with example metrics.

as follows. Section 2 formally defines AveP, $\mathrm{Q}$-measure, RR and O-measure. Section 3 describes the methods we use for comparing the metrics in terms of resemblance, stability and discrimination power in system ranking. Section 4 describes the data from NTCIR-3 used in our analyses. Section 5 compares the four metrics in terms of system ranking resemblance. Sections 6 and 7 compare the metrics in terms of stability and discrimination power, respectively. Finally, Section 8 concludes this paper. In addition, the Appendix contains some theorems and proofs for O-measure and Q-measure.

\section{Metrics}

\subsection{Average Precision}

AveP is an IR metric based on binary relevance, designed for the task of finding all relevant documents with high precision. It is perhaps the most widely-used IR metric today.

Let $R$ denote the total number of relevant documents for a topic, and let count $(r)$ denote the number of relevant documents within top $r$ of the ranked output. Then, clearly, Precision at Rank $r$ is given by

$$
P(r)=\frac{\operatorname{count}(r)}{r}
$$

Let $i \operatorname{srel}(r)$ denote a flag, such that $i s r e l(r)=1$ if the document at Rank $r$ is relevant and $\operatorname{isrel}(r)=0$ otherwise. Then, AveP can be expressed as:

$$
\text { AveP }=\frac{1}{R} \sum_{1 \leq r \leq L} i \operatorname{srel}(r) P(r)
$$

where $L$ is the ranked output size. (Following the TREC/NTCIR traditions, we let $L \leq L^{\prime}=$ 1000 throughout this paper.)

As is described in Section 4, the NTCIR test collections have graded relevance assessments. Throughout this paper, we use the Relaxed version of $\mathrm{AveP}^{2)}$, which treats all S-relevant (i.e., highly relevant), A-relevant (i.e., relevant) and B-relevant (i.e., partially relevant) documents as just "relevant". Thus, $R(S)+R(A)+$ $R(B)=R$ holds for each topic where $R(X)$ denotes the number of $X$-relevant documents.

\subsection{Q-measure}

Q-measure, first proposed at the NTCIR-4 Workshop, is an IR metric based on graded relevance, designed for the task of finding all relevant documents with high precision. It is very highly correlated with AveP, and is at least as stable and sensitive as $\mathrm{AveP}^{8), 10), 13)}$.

Let $X$ denote a relevance level, and let gain $(X)$ denote the gain value (i.e., "reward") for successfully retrieving an $X$-relevant document. As $X \in\{S, A, B\}$ for the NTCIR CLIR tracks, we use $\operatorname{gain}(S)=3$, gain $(A)=2$ and $\operatorname{gain}(B)=1$ by default. The gain at Rank $r$ is defined as $g(r)=\operatorname{gain}(X)$ if the document at Rank $r$ is $X$-relevant and $g(r)=0$ if it is nonrelevant. Moreover, the cumulative gain ${ }^{6), 7)}$ at Rank $r$ is defined as:

$$
c g(r)=\sum_{1 \leq i \leq r} g(i)
$$

In particular, let $g_{I}(r)$ and $c g_{I}(r)$ represent the (cumulative) gain at Rank $r$ for an ideal ranked output, which has all the S-relevant documents at the top of the list, followed by all A-relevant ones, followed by all B-relevant ones in the case of NTCIR. (A more formal definition can be found in the Appendix.) Then, Q-measure can be expressed as:

$$
Q \text {-measure }=\frac{1}{R} \sum_{1 \leq r \leq L} i \operatorname{srel}(r) B R(r)
$$

where

$$
B R(r)=\frac{c g(r)+\operatorname{count}(r)}{c g_{I}(r)+r} .
$$

We call $B R(r)$ the blended ratio at Rank $r$.

The Appendix contains proofs that Qmeasure has the following properties:

- $Q$-measure $=1$ if and only if the system output is an ideal one ${ }^{8)}$.

- In a binary relevance environment, $Q$-measure $=A v e P$ holds if and only if there is no relevant document below Rank $R$.

- In a binary relevance environment, $Q$-measure $>$ AveP holds if and only if there is at least one relevant document below Rank $R$. 
Table 1 O-measure values for systems that return exactly one relevant document for a topic s.t. $R(S)=1, R(A)=1, R(B)=1$.

\begin{tabular}{l|l|l|l|l}
\hline \hline & Relaxed AveP & Q-measure & RR & O-measure \\
\hline (a) S at Rank 1 & $0.3333(=(1 / 1) / 3)$ & $0.3333(=(4 / 4) / 3)$ & $1(=1 / 1)$ & $1(=4 / 4)$ \\
(b) A at Rank 1 & $0.3333(=(1 / 1) / 3)$ & $0.2500(=(3 / 4) / 3)$ & $1(=1 / 1)$ & $0.7500(=3 / 4)$ \\
(c) B at Rank 1 & $0.3333(=(1 / 1) / 3)$ & $0.1667(=(2 / 4) / 3)$ & $1(=1 / 1)$ & $0.5000(=2 / 4)$ \\
(d) S at Rank 2 & $0.1667(=(1 / 2) / 3)$ & $0.1905(=(4 / 7) / 3)$ & $0.5000(=1 / 2)$ & $0.5714(=4 / 7)$ \\
(e) A at Rank 2 & $0.1667(=(1 / 2) / 3)$ & $0.1429(=(3 / 7) / 3)$ & $0.5000(=1 / 2)$ & $0.4286(=3 / 7)$ \\
(f) B at Rank 2 & $0.1667(=(1 / 2) / 3)$ & $0.0952(=(2 / 7) / 3)$ & $0.5000(=1 / 2)$ & $0.2857(=2 / 7)$ \\
\hline
\end{tabular}

\subsection{Reciprocal Rank}

$\mathrm{RR}$ can be regarded as an IR metric based on binary relevance for the task of finding one relevant document with high precision. If the system output does not contain a relevant document, we let $R R=0$. Otherwise, we let $R R=1 / r^{\prime}=P\left(r^{\prime}\right)$, where $r^{\prime}$ is the rank of the retrieved relevant document that is nearest to the top of the list.

Clearly,

- If $R=1$, then $R R=A v e P$.

Thus, while AveP looks at the Precision for every relevant document, RR looks at the Precision for the first relevant one only. This makes $\mathrm{RR}$ a relatively unstable measure, and therefore larger topic sets should be used in experiments in order to obtain reliable results.

\subsection{O-measure}

We now define O-measure, an IR metric based on graded relevance, designed for the task of finding One relevant document with high precision. If the system output does not contain a relevant document, we let $O$-measure $=0$. Otherwise,

$$
\begin{aligned}
\text { O-measure } & =B R\left(r^{\prime}\right) \\
& =\frac{c g\left(r^{\prime}\right)+\operatorname{count}\left(r^{\prime}\right)}{c g_{I}\left(r^{\prime}\right)+r^{\prime}} \\
& =\frac{g\left(r^{\prime}\right)+1}{c g_{I}\left(r^{\prime}\right)+r^{\prime}} .
\end{aligned}
$$

Clearly,

- If $R=1$, then $O$-measure $=Q$-measure.

Thus, while Q-measure looks at the blended ratio for every relevant document, O-measure looks at that of the first relevant one only.

Intuitively, O-measure compares the system output with the ideal one in terms of cumulative gain just when the first retrieved relevant document is found, but makes sure that "late arrival" of a relevant document is penalised (See Section 2.5).

Table 1 compares O-measure with other metrics for six imaginary systems (a)-(f) that return exactly one relevant document for a topic s.t. $R(S)=R(A)=R(B)=1$. Thus, with default gain values, the sequence of $g_{I}$ for this topic is $(3,2,1,0, \ldots)$ and that of $c g_{I}(r)$ is $(3,5,6,6, \ldots)$. System (e), for example, has one A-relevant document at Rank $2\left(r^{\prime}=2\right)$, so $O$-measure $=(g(2)+1) /\left(c g_{I}(2)+2\right)=$ $(2+1) /(5+2)=3 / 7$. It can be observed that, unlike RR, O-measure can discriminate among Systems (a)-(f) using graded relevance. Note that, with default gain values, both Q-measure and O-measure judge that System (d) ("S at Rank 2") is better than (c) ("B at Rank 1"), disagreeing with both AveP and RR. With a different gain value assignment, we can get a different system ranking. Ideally, gain values should be assigned so that the metrics correlate well with user satisfaction, but this issue is beyond the scope of this paper.

An important assumption underlying Omeasure is that the user is expected to stop examining the ranked list as soon as he finds any relevant document. That is, even a partially relevant document would be okay for him. But consider a ranked output that has a B-relevant document at Rank 2 and an S-relevant document at Rank 3. O-measure ignores the S-relevant document completely. We argue that this is an acceptable model of some IR environments: For example, the ranked list presented to the user may be uninformative (with vague document titles, low-quality keyword-incontext text snippets, etc.), so that it is difficult for him to immediately judge the relevancy or the relevance grade of each document; Alternatively, the user may be using an IR interface that presents the retrieved documents one by one without ever showing a ranked list, in which case the user is never aware of what is coming next until he presses the "show next document" button. In these situations, the user may well be content with the B-relevant document at Rank 2, never knowing that there is a much better document at Rank 3 .

On the other hand, for IR environments in which it would be easy for the user to see beyond the B-relevant document at Rank 2 and 
to realise that there is an S-relevant document at Rank 3, we can use yet another variant of $\mathrm{Q}$-measure/O-measure ${ }^{14)}$. In this paper, we use O-measure as a tool for studying the properties of Task D shown in Fig. 1, rather than claiming that it is the only metric conceivable for the task.

The Appendix contains proofs that Omeasure has the following properties:

- O-measure $=1$ if and only if the system output has any one of the most relevant documents at Rank 1.

- If a system output contains its first relevant document at Rank $r^{\prime}$ in a binary relevance environment, $O$-measure $=R R$ holds if and only if $r^{\prime} \leq R$.

- If a system output contains its first relevant document at Rank $r^{\prime}$ in a binary relevance environment, $O$-measure $>R R$ holds if and only if $r^{\prime}>R$.

Following the discussion of the relationship between AveP and Q-measure ${ }^{10)}$, it can also be observed that:

- If small gain values (e.g., gain $(S)=0.3$, $\operatorname{gain}(A)=0.2, \operatorname{gain}(B)=0.1)$ are used, the values of $g\left(r^{\prime}\right)$ and $c g_{I}\left(r^{\prime}\right)$ would also be small and therefore O-measure would resemble RR (See Eq. (8)).

Just like RR, O-measure is probably a relatively unstable metric since it relies on the first retrieved relevant document only. However, as we shall see in Section 6, it may be more stable than RR as it exploits graded relevance.

\subsection{What about Other Metrics?}

One necessary condition for a reliable IR metric is that it defines the same performance upperbound (usually 1.0) for every topic, so that averaging across topics becomes valid. Metrics such as Precision at a fixed document cut-off and unnormalised (discounted) cumulative gain ${ }^{6), 7)}$ do not satisfy this condition ${ }^{13)}$. Even with normalisation, naive metrics such as $c g\left(r^{\prime}\right) / c g_{I}\left(r^{\prime}\right)$ would not work: For example, consider a topic s.t. $R=R(B)=3$, so that its sequence of $c g_{I}(r)$ is $(1,2,3,3, \ldots)$. Suppose that System $x$ retrieves one B-relevant document at Rank $3\left(r^{\prime}=3\right)$, whereas System $y$ retrieves one B-relevant document at Rank $1000\left(r^{\prime}=1000\right)$. Even though System $x$ is clearly superior to System $y$, they would receive the same score, namely $1 / 3$. This is because $c g_{I}(r)$ freezes after Rank $R$ (See the Appendix, Eq. (13)). Using discounted cumulative gains ${ }^{6), 7)}$ can also penalise late arrival of relevant documents, but this would require an additional parameter, namely the logarithm base $^{6), 7)}$. Note also that the use of the logarithm makes it difficult to calculate the metric values by hand.

At the NTCIR-3 Web track, Weighted Reciprocal Rank (WRR) was proposed for handling graded relevance, which, in theory, is a kind of generalised $\mathrm{RR}^{5)}$. However, in practice, the parameters of WRR are chosen so that it is only equivalent to traditional RR. Specifically, the NTCIR Web track uses two versions of traditional RR: one based on all relevant documents (the "Relaxed RR"), and the other computed after excluding partially relevant ones (the "Rigid" RR).

\section{Metrics Comparison Methods}

This section describes the methods we use for comparing the metrics in terms of resemblance, stability and discrimination power in system ranking.

\subsection{Resemblance with Other Metrics in System Ranking}

The first method we use for comparing IR metrics is to examine how the rankings of the systems that participated in a common IR task resemble each other. For this purpose, we compute two versions of rank correlations: Spearman's (e.g., 3)) and Kendall's (e.g., 18)). Rank correlations are based on how many swaps occur between two rankings and lie between -1 and 1 , where 1 implies that the rankings are identical while -1 implies that the rankings are completely reversed. It is also known that the Kendall's rank correlations are lower than Spearman's for most data.

For the purpose of comparing IR metrics, however, we believe that the discussion of rank correlations is not enough. This is because rank correlations do not tell us at which ranks the swaps occur. For IR evaluations, swaps in the top ranks may be more important than those in the lowest ranks. Thus, in addition to examining rank correlations, we visualise the system swaps and examine specific system pairs, as well as specific topics, where necessary.

\subsection{Stability with Respect to Change in the Topic Set}

The second method we use for comparing IR metrics is to examine the stability with respect to change in the topic set, using our adaptation of the Buckley/Voorhees method ${ }^{1)}$. The input to the Buckley/Voorhees method are: 
for $i=1$ to 1000

create $Q_{i} \subset Q$ at random s.t. $\left|Q_{i}\right|==c$;

for each pair of systems $x, y \in S$

for $i=1$ to 1000

$$
\begin{aligned}
& \operatorname{margin}=f * \max \left(M\left(x, Q_{i}\right), M\left(y, Q_{i}\right)\right) \\
& \operatorname{if}\left(\left|M\left(x, Q_{i}\right)-M\left(y, Q_{i}\right)\right|<\text { margin }\right) \\
& \quad E Q_{M}(x, y)++ \\
& \text { else } \operatorname{if}\left(M\left(x, Q_{i}\right)>M\left(y, Q_{i}\right)\right) \\
& \quad G T_{M}(x, y)++ \\
& \text { else } \\
& \qquad G T_{M}(y, x)++
\end{aligned}
$$

Fig. 2 The algorithm for computing $E Q_{M}(x, y)$, $G T_{M}(x, y)$ and $G T_{M}(y, x)$.

- An IR test collection;

- A set of systems (or runs) submitted to the task defined by the above test collection;

- The topic set size $c$ (See later);

- An IR evaluation metric;

- A fuzziness value $f$, which determines how much relative performance difference between a system pair should be regarded as negligible.

The output of the method are:

- Minority Rate, which represents the lack of stability with respect to change in the topic set;

- Proportion of Ties, which represents the lack of discrimination power.

More specifically, the Buckley/Voorhees method works as follows. Let $S$ denote a set of systems submitted to a particular task, and let $x$ and $y$ denote a pair of systems from $S$. Let $Q$ denote the entire set of topics used in the task, and let $c$ be a constant. Let $M\left(x, Q_{i}\right)$ denote the value of metric $M$ for System $x$ calculated based on a topic set $Q_{i}(\subset Q)$. Then, using the algorithm shown in Fig. 2, the minority rate and the proportion of ties of $M$, given a fuzziness value $f$, can be computed as:

$$
\begin{aligned}
& \text { MinorityRate }_{M}= \\
& \frac{\sum_{x, y \in S} \min \left(G T_{M}(x, y), G T_{M}(y, x)\right)}{\sum_{x, y \in S} 1000} . \\
& \text { Prop Ties }_{M}= \\
& \frac{\sum_{x, y \in S} E Q_{M}(x, y)}{\sum_{x, y \in S} 1000} .
\end{aligned}
$$

From the algorithm, it is clear that $G T_{M}(x, y)+G T_{M}(y, x)+E Q_{M}(x, y)=1000$ for each system pair, and that a larger $f$ yields larger $E Q_{M}(x, y)$ values, and therefore a larger proportion of ties and a smaller minority rate. That is, there is a trade-off between these two statistics. As a fixed value of $f$ implies different trade-offs for different metrics, we vary $f(=0.01,0.02, \ldots, 0.10)$ and draw minorityrate / proportion-of-ties curves ${ }^{9), 13)}$ for the purpose of comparing different metrics.

\subsection{Discrimination Power given a Con- fidence Level}

The third method we use for comparing IR metrics is to examine the discrimination power using our adaptation of the Voorhees/Buckley method ${ }^{19)}$. The input to the Voorhees/Buckley method are:

- An IR test collection;

- A set of systems submitted to the task defined by the above test collection;

- The topic set size $c$ (See later);

- An IR evaluation metric;

- The required confidence level of a conclusion as to which of the given two systems $x$ and $y$ is better.

The output of the method are:

- The mininum absolute/relative performance difference required in order to guarantee the given confidence level;

- How often system pairs actually satisfy the above requirement, which represents the discrimination power of the metric.

More specifically, the Voorhees/Buckley method works as follows. Let $d$ denote a performance difference between two systems computed based on a topic set. We first prepare 21 performance difference bins, where the first bin represents performance differences such that $0 \leq d<0.01$, the second bin represents those such that $0.01 \leq d<0.02$, and so on, and the last bin represents those such that $0.20 \leq d$. Let $B I N(d)$ denote a mapping from a difference $d$ to one of the 21 bins where it belongs. The algorithm shown in Fig. 3 calculates a swap rate for each bin. (Our test is stricter than the original one by Voorhees/Buckley, in that our "swaps" include cases where only one of $d_{M}\left(Q_{i}\right)$ and $d_{M}\left(Q_{i}^{\prime}\right)$ is zero: This is because Voorhees/Buckley's original test, which increments the swap count only when $d_{M}\left(Q_{i}\right) * d_{M}\left(Q_{i}^{\prime}\right)<0$, tends to underrate the swap rates for near-zero bins as the differences are actually quite often zero. We have verified that this modification gives graphs that look more stable, but do not affect our conclusions.)

By plotting swap rates against the performance difference bins, one can discuss how much performance differences are required in 


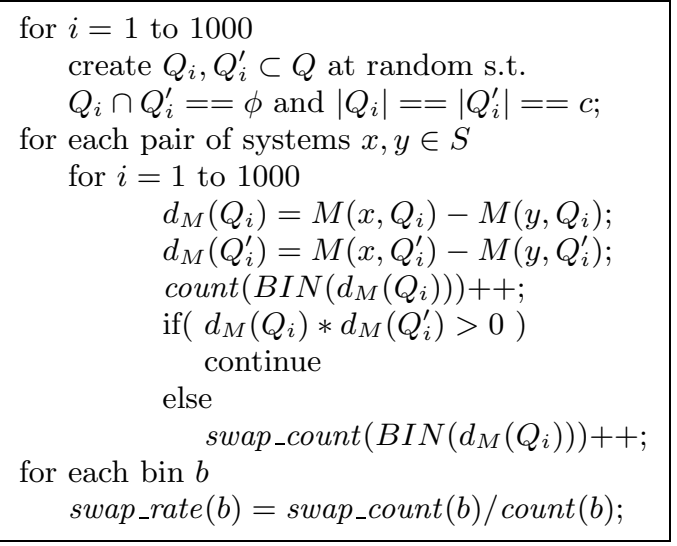

Fig. 3 The algorithm for computing the swap rates.

order to conclude that a run is better than another with a required confidence level. For example, if $95 \%$ confidence is required, one looks for the minumum performance difference that guarantees $5 \%$ swap rate or less. Moreover, by examining how often this condition is satisfied among all pairwise comparisons from all the trials, one can compare the discrimination power of different metrics.

Because $Q_{i}$ and $Q_{i}^{\prime}$ must be disjoint, they can only be up to half the size of the original topic set $Q$. Voorhees/Buckley have used extrapolation for larger topic set sizes, but we stick to the statistics actually measured in our study, since our goal is to compare different metrics under the same condition.

Recently, Sanderson and Zobel ${ }^{15)}$ claimed that the Voorhees/Buckley method overestimates the swap rates due to sampling without replacement from the original topic set $Q$. What they see as a problem is the fact that $Q_{i}$ and $Q_{i}^{\prime}$ are not independent of each other as they are disjoint: they claim that the Voorhees/Buckley method yields swap rate upperbounds. Based on this observation, they used sampling with replacement instead to provide swap rate lowerbounds. However, more recently, Sakai ${ }^{11)}$ showed that sampling with and without replacement yield similar results for relative comparisons of different IR metrics. This paper therefore discusses the withoutreplacement results only: the corresponding with-replacement results, which are very similar to the ones reported in this paper, can be found in Ref. 11).

\section{Data}

In our experiments, we used the Chinese, Japanese, English and Korean document runs from the NTCIR-3 CLIR tasks, which are known as the C-, J-, E- and K-runs, respectively ${ }^{10)}$. Specifically, the following files, provided by National Institute of Informatics, Japan, were used for the analyses:

- ntc3clir-allCruns.20040511.zip (45 Runs for retrieving Chinese documents: "C-runs")

- ntc3clir-allJruns.20040511.zip

(33 Runs for retrieving Japanese documents: "J-runs")

- ntc3clir-allEruns.20040511.zip

(24 Runs for retrieving English documents: "E-runs")

- ntc3clir-allKruns.20040511.zip

(14 Runs for retrieving Korean documents: "K-runs")

The above files contain runs submitted by 14 different participants, and include both monolingual and cross-language runs, as well as runs using different topic fields, e.g., TITLE, DESCRIPTION etc. (There were 23 participants at the NTCIR-3 CLIR Task, but not all of them have agreed to the release of their submission files.)

Table 2 shows the number of topics and the number of relevant documents per topic for each of the four document collections. Among the four sets of data (i.e., test collections plus submitted runs), the Chinese and the Japanese data are probably more reliable than the rest because they contain over 30 runs and over 40 topics. Note also that there are relatively few S-relevant documents for the Japanese case.

Other NTCIR data such as those from the Web track are currently not available to us. Even if they were, the limited number of runs will make it difficult to conduct reliable experiments. We hope that these problems will be resolved with the future growth of NTCIR.

\section{System Ranking Resemblance}

This section examines how the system rankings using (Relaxed) AveP ${ }^{10)}$, Q-measure, RR and $\mathrm{O}$-measure resemble each other, using the four data sets described earlier.

\subsection{Rank Correlations}

Table 3 shows the Spearman/Kendall rank correlations of the system rankings using AveP, Q-measure, RR and O-measure for the four 
Table 2 Number of relevant documents per topic in NTCIR-3.

\begin{tabular}{l|r|r|r|r|r}
\hline \hline & \#topics & $R$ & $R(S)$ & $R(A)$ & $R(B)$ \\
\hline $\mathrm{C}$ & 42 & 78.2 & 21.0 & 24.9 & 32.3 \\
$\mathrm{~J}$ & 42 & 60.4 & 7.9 & 31.5 & 21.0 \\
$\mathrm{E}$ & 32 & 23.2 & 3.6 & 10.2 & 9.3 \\
$\mathrm{~K}$ & 30 & 128.9 & 32.9 & 36.5 & 59.5 \\
\hline
\end{tabular}

Table 3 Spearman/Kendall rank correlations for NTCIR-3 CLIR formal runs.

\begin{tabular}{|c|c|c|c|}
\hline & Q-measure & $\mathrm{RR}$ & O-measure \\
\hline \multicolumn{4}{|l|}{ (a) 45 C-runs } \\
\hline Relaxed AveP & $.9982 / .9798$ & $.8567 / .6990$ & $.8411 / .6929$ \\
\hline Q-measure & - & $.8594 / .7030$ & $.8464 / .6929$ \\
\hline $\mathrm{RR}$ & - & - & $\mathbf{. 9 7 4 4 / . 8 8 4 8}$ \\
\hline \multicolumn{4}{|l|}{ (b) 33 J-runs } \\
\hline Relaxed AveP & $.9947 / .9583$ & $\mathbf{. 9 3 2 8 / . 7 9 9 2}$ & $\mathbf{. 9 0 4 7 / . 7 4 6 2}$ \\
\hline Q-measure & - & $\mathbf{. 9 3 6 5 / . 8 0 3 0}$ & $\mathbf{. 9 1 4 4 / . 7 5 7 6}$ \\
\hline $\mathrm{RR}$ & - & - & $\mathbf{. 9 6 1 6 / . 8 4 0 9}$ \\
\hline \multicolumn{4}{|l|}{ (c) 24 E-runs } \\
\hline Relaxed AveP & $.9974 / .9783$ & $\mathbf{. 9 1 7 4 / . 7 6 0 9}$ & $\mathbf{. 9 2 6 1 / . 7 8 2 6}$ \\
\hline Q-measure & - & $\mathbf{. 9 1 7 4 / . 7 5 3 6}$ & $\mathbf{. 9 2 3 5 / . 7 7 5 4 ~}$ \\
\hline $\mathrm{RR}$ & - & - & $.9861 / .9203$ \\
\hline \multicolumn{4}{|l|}{ (d) 14 K-runs } \\
\hline Relaxed AveP & $.9912 / .9560$ & $.9560 / .8681$ & $\mathbf{. 9 5 1 6 / . 8 4 6 2}$ \\
\hline Q-measure & - & $\mathbf{. 9 4 7 3 / . 8 2 4 2}$ & $\mathbf{. 9 5 1 6 / . 8 4 6 2}$ \\
\hline $\mathrm{RR}$ & - & - & $.9956 / .9780$ \\
\hline
\end{tabular}

Table 4 Spearman/Kendall rank correlations for NTCIR-3 CLIR formal runs with different gain values.

\begin{tabular}{|c|c|c|c|c|}
\hline & O30:20:10 & O0.3:0.2:0.1 & O1:1:1 & O10:5:1 \\
\hline \multicolumn{5}{|c|}{ (a) 45 C-runs } \\
\hline $\mathrm{RR}$ & $\mathbf{. 9 5 7 4 / . 8 3 8 4}$ & $.9934 / 9434$ & $.9999 / .9980$ & $\mathbf{. 9 3 9 9 / . 8 0 0 0}$ \\
\hline O-measure & $.9949 / .9535$ & $.9897 / .9374$ & $\mathbf{. 9 7 5 4 / . 8 8 6 9}$ & $.9866 / .9152$ \\
\hline \multicolumn{5}{|c|}{ (b) 33 J-runs } \\
\hline $\mathrm{RR}$ & $\mathbf{. 9 5 5 5 / . 8 2 2 0}$ & $.9876 / .9394$ & $1.0000 / 1.0000$ & $\mathbf{. 9 3 1 8 / . 7 7 2 7}$ \\
\hline O-measure & $.9970 / .9735$ & $.9803 / .9015$ & $.9616 / .8409$ & $.9876 / .9318$ \\
\hline \multicolumn{5}{|c|}{ (c) 24 E-runs } \\
\hline $\mathrm{RR}$ & $.9757 / .9058$ & $.9957 / .9710$ & $.9991 / .9928$ & $.9536 / .8406$ \\
\hline O-measure & $.9922 / .9565$ & $.9922 / .9493$ & $.9870 / .9275$ & $.9748 / .9058$ \\
\hline \multicolumn{5}{|c|}{ (d) 14 K-runs } \\
\hline $\mathrm{RR}$ & $.9868 / .9560$ & $1.0000 / 1.0000$ & $1.0000 / 1.0000$ & $.9868 / .9560$ \\
\hline O-measure & $.9956 / .9780$ & $.9956 / .9780$ & $.9956 / .9780$ & $.9956 / .9780$ \\
\hline
\end{tabular}

data sets ${ }^{\text {z }}$. For example, Table 3 (a) shows that the Spearman/Kendall rank correlations between RR and O-measure are .9744/.8848, respectively. Table 4 focuses on $\mathrm{RR}$ and O-measure (i.e., two metrics designed for finding one relevant document), and shows how changing the gain values affect rank correlations. For example, "O10:5:1" represents O-measure with $\operatorname{gain}(S)=10, \operatorname{gain}(A)=5$, $\operatorname{gain}(B)=1$.

\footnotetext{
is Similar tables we presented at FIT 2005 12) contained some incorrect Kendall correlation values. As a result, we underestimated the robustness of $\mathrm{O}$-measure with respect to gain value assignment.
}

From the two tables, it can be observed that:

- The correlations between AveP/Q-measure and RR/O-measure are relatively low. For example, the Kendall correlation between AveP and RR for the C-runs is only .6990. This confirms that systems that can find many relevant documents with high precision are not necessarily those that can find one relevant document with high precision. Although this may not sound like a novel finding, note that we have provided the evidence not only for binary relevance but also for graded relevance.

- The correlations between RR and 


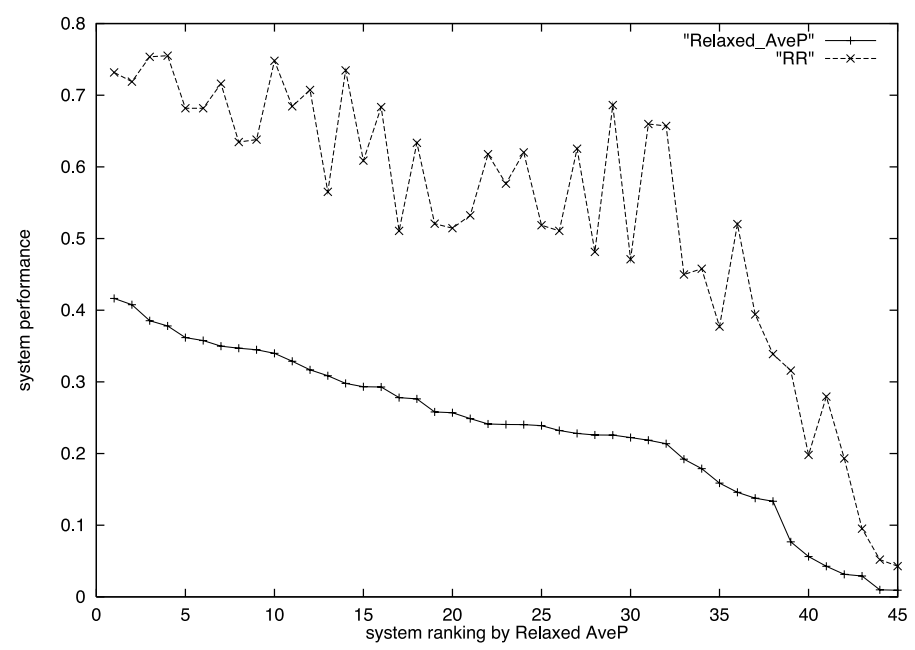

Fig. 4 System ranking: AveP vs. RR (45 C-runs).

O-measure are not always high. For example, the Kendall correlation between RR and O-measure for the J-runs is only .8409, and that between RR and O10:5:1 for the J-runs is only .7727. This suggests that systems that can find one highly relevant document with high precision are not necessarily those that can find any one relevant document with high precision.

- Both "flat" gain values (O1:1:1) and small gain values (O0.3:0.2:0.1) make O-measure resemble $\mathrm{RR}$, confirming the theoretical arguments in Section 2.4.

- O-measure is quite robust to the choice of gain values, since the correlations between the default O-measure and nondefault ones are all very high. That is, the system ranking is quite stable with respect to change in gain values.

Thus, for the task of finding one highly relevant document with high precision, we would recommend $\mathrm{O}$-measure with small gain values (e.g., O0.3:0.2:0.1), since this gives high correlations with traditional $\mathrm{RR}$ and can take graded relevance into account.

As was mentioned earlier, the rank correlations disregard whether a swap occurs near the top of the ranked system list or near the bottom. Below, we therefore take a closer look at the resemblance of system rankings using the Chinese data set which is probably the most reliable.

\subsection{AveP versus RR}

Figure 4 visualises the ranking disagreements between AveP and RR for the C-runs, which were sorted by AveP and then numbered from 1 to 45. Thus, an increase in the RR curve represents a swap compared to the the AveP ranking. For example, the AveP values of the 13th/14th runs in this graph (hereafter refered to as Runs $x$ and $y$ ) are $0.3086 / 0.2979$, while the $\mathrm{RR}$ values are $0.5651 / 0.7347$, respectively.

Figure 5 compares Run $x$ and Run $y$ pertopic, where the vertical axis shows the performance of $x$ minus that of $y$. It can be observed that there are disagreements between AveP and $\mathrm{RR}$ even on a per-topic basis. For example, for Topic $027, x$ slightly outperforms $y$ in terms of AveP while $y$ outperforms $x$ by 0.5 in terms of RR. These analyses also confirm that finding one relevant document and finding all relevant documents are different tasks.

\subsection{RR versus $\mathrm{O}$-measure}

Figure 6 visualises the ranking disagreements between $\mathrm{RR}$ and O-measure for the C-runs, which were sorted this time by RR and then numbered from 1 to 45. Many ranking disagreements are visible, due to the introduction of graded relevance. For example, the RR values of the 5 th/6th runs in this graph (hereafter referred to as Runs $z$ and $w$ ) are $0.7318 / 0.7190$, while the O-measure values are $0.6140 / 0.6483$, respectively. We compared Run $z$ and Run $w$ per-topic, and found that $\mathrm{RR}$ and $\mathrm{O}$-measure disagrees with each other for Topic 050: For this topic, $R(S)=12$, and Run $z$ retrieved a B-relevant document at Rank 7 while Run $w$ retrieved an S-relevant document at Rank 8. Thus, $z$ outperforms $w$ in terms of $\mathrm{RR}$, but $w$ outperforms $z$ in terms of O-measure, having been rewarded for finding a highly relevant document. (O-measure for $z$ is $(1+1) /(3 * 7+7)=$ 


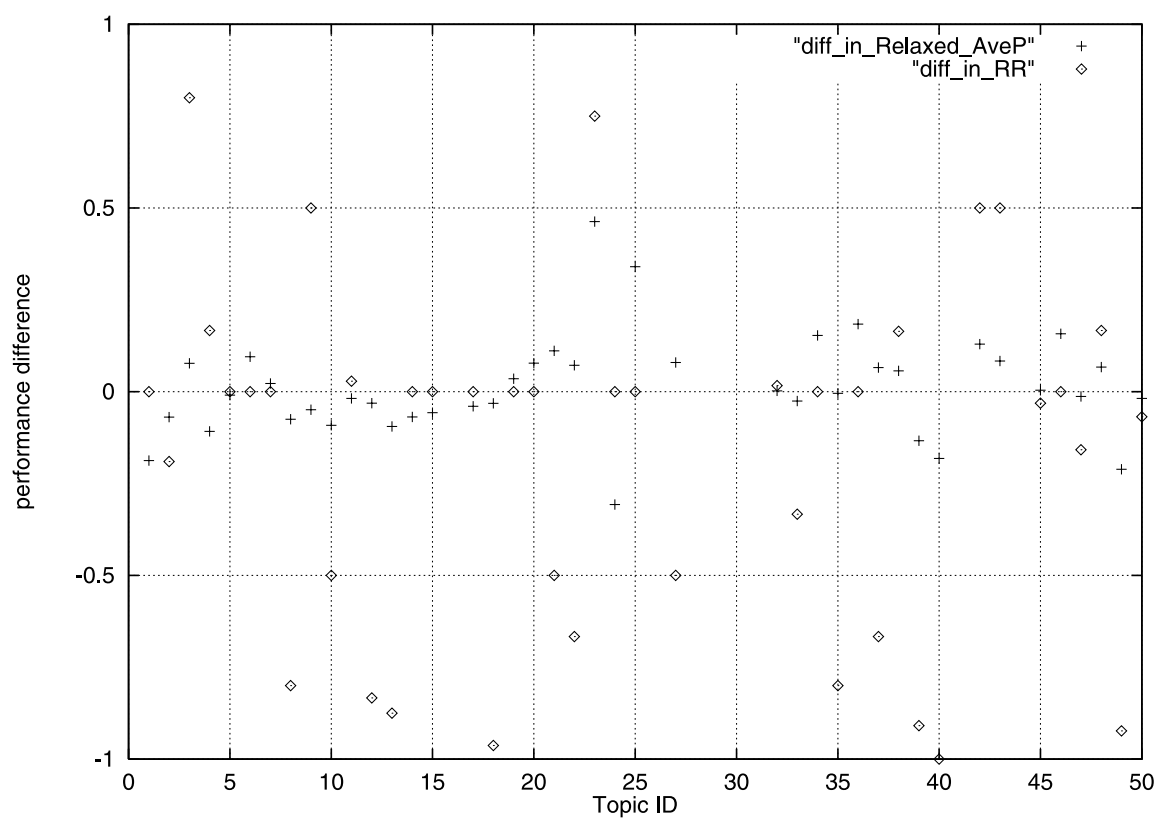

Fig. 5 Per-topic comparison of Run $x$ and Run $y$ (13th and 14th best runs in terms of Relaxed AveP).

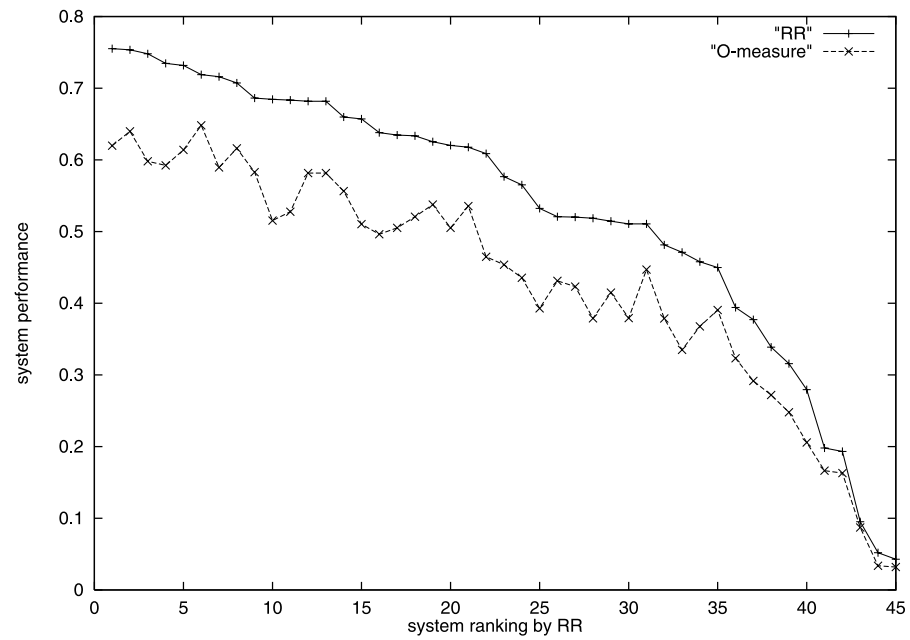

Fig. 6 System ranking: RR vs. O-measure (45 C-runs).

0.0714 , while that for $w$ is $(3+1) /(3 * 8+8)=$ 0.1250 .) These analyses also suggest that finding one highly relevant document and finding any one relevant document are different tasks.

\section{System Ranking Stability}

This section examines the stability of AveP, Q-measure, RR and O-measure using our adaptation of the Buckley/Voorhees method. We focus on the two most reliable data sets from NTCIR-3 CLIR, namely, the Chinese and Japanese ones. With each data set, we use the top 30 runs for computing minority rates and proportion of ties. Our stability experiments share 1,000 topic sets $Q_{i}$ drawn from $Q$ with our discrimination power experiments reported in Section 7: since the latter requires that $\left|Q_{i}\right| \leq|Q| / 2$ (See Section 3.3), and we have 42 Chinese and 42 Japanese topics, we use $c=\left|Q_{i}\right|=20$ throughout this paper.

Figures $\mathbf{7}$ and $\mathbf{8}$ show the trade-offs between the minority rate and proportion of ties computed using the Chinese and the Japanese data, respectively. Recall that stable metrics 


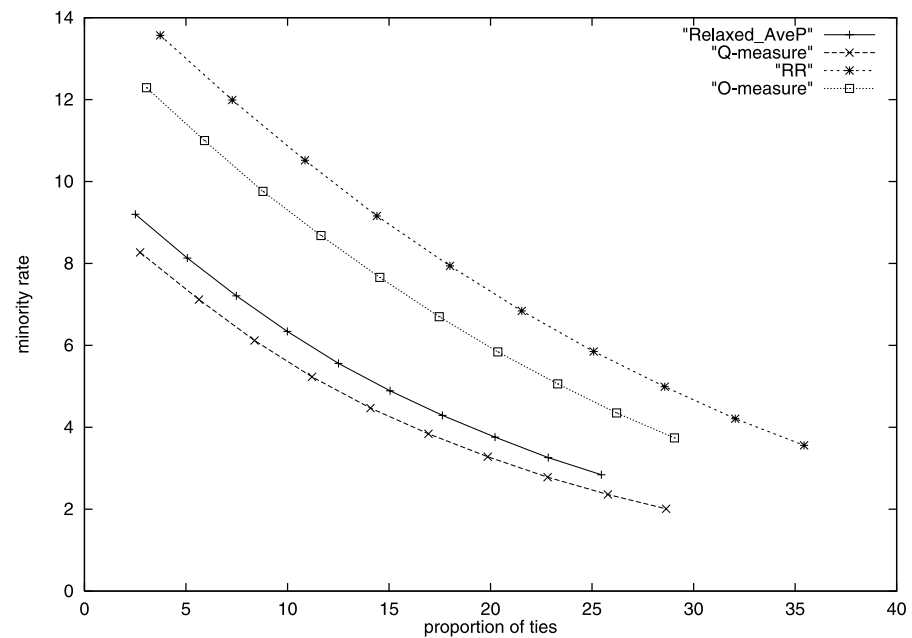

Fig. 7 Minority rate/proportion of ties (Top 30 C-runs; 20 topics).

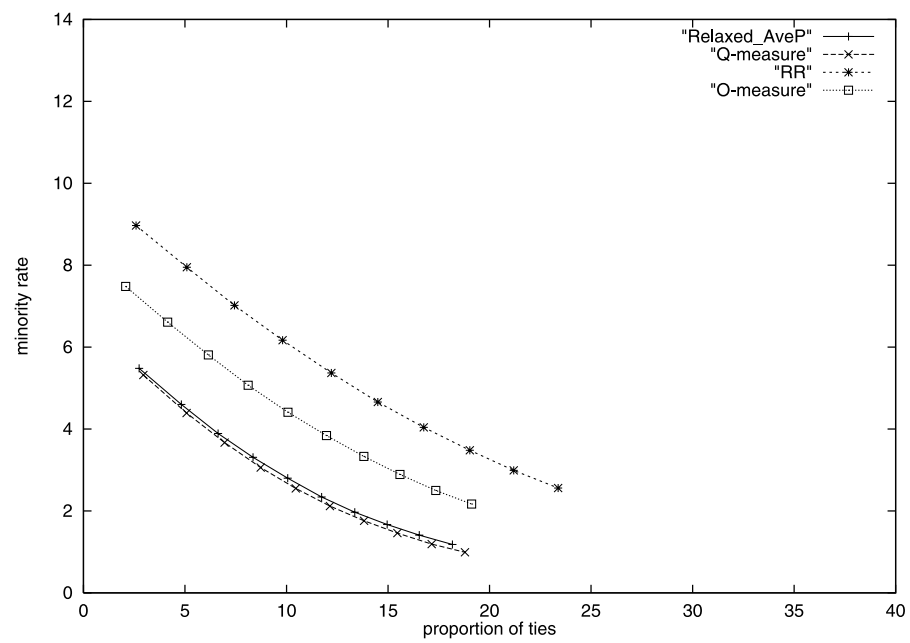

Fig. 8 Minority rate/proportion of ties (Top 30 J-runs; 20 topics).

are those that show low minority rates and low proportion of ties. Our findings are:

- For both of the data sets, AveP and Q-measure are clearly more stable than RR and O-measure. In other words, examining how all of the relevant documents are retrieved gives more stability than examining how one relevant document is retrieved, in both binary and graded relevance environments.

- For both of the data sets, O-measure is more stable than RR, suggesting that the use of graded relevance may help stabilise evaluation.

\section{System Ranking Sensitivity}

This section examines the discrimination power (or sensitivity) of AveP, Q-measure, RR and O-measure using our adaptation of the Voorhees/Buckley method. The data sets used for this set of experiments are the same as those for our stability experiments, except that another set of 1,000 topic sets $Q_{i}^{\prime}$, each of which is compared with $Q_{i}$, is used (See Section 3.3).

Table 5 summarises the results of our experiments using our adaptation of the Voorhees/Buckley method with the Chinese data. Column (i) shows the absolute performance differences required to guarantee a given confidence level, and Column (ii) shows the maximum mean performance observed among all combinations of runs and topic subsets generated for the experiment. (Since there were 30 runs and 2,000 topic sets, there were 60,000 mean performance values in total.) Column (iii) is just Column (i) divided by (ii), that 
Table 5 Discrimination power of metrics (Top 30 C-runs; 20 topics).

\begin{tabular}{l|r|r|r|r}
\hline \hline & $\begin{array}{r}\text { (i) absolute diff } \\
\text { required }\end{array}$ & $\begin{array}{r}\text { (ii) max } \\
\text { performance } \\
\text { observed }\end{array}$ & $\begin{array}{r}\text { (iii) relative diff } \\
\text { required }\end{array}$ & $\begin{array}{r}\text { (iv) \%comparisons } \\
\text { with }\end{array}$ \\
\hline (a) 95\% confidence (5\% swap rate) \\
\hline Relaxed AveP
\end{tabular}

Table 6 Discrimination power of metrics at 95\% confidence (Top 30 J-runs; 20 topics).

\begin{tabular}{l|r|r|r|r}
\hline \hline & $\begin{array}{r}\text { (i) absolute diff } \\
\text { required }\end{array}$ & $\begin{array}{r}\text { (ii) max } \\
\text { performance } \\
\text { observed }\end{array}$ & $\begin{array}{r}\text { (iii) relative diff } \\
\text { required }\end{array}$ & $\begin{array}{r}\text { (iv) \%comparisons } \\
\text { with }\end{array}$ \\
\hline Relaxed AveP & 0.11 & .6305 & $17 \%$ & $51.7 \%$ \\
Q-measure & 0.12 & .6354 & $18 \%$ & $49.0 \%$ \\
RR & 0.20 & .9750 & $20 \%$ & $41.2 \%$ \\
O-measure & 0.20 & .8950 & $22 \%$ & $41.4 \%$ \\
\hline
\end{tabular}

is, how the absolute difference translates to relative difference required. Column (iv) shows how many of the system pair comparisons actually satisfied the requirement: That is, it represents the discrimination power of the metric. (There were $30 * 29 / 2=435$ system pairs, each with 1,000 trials, and therefore the number of comparisons considered here is 435,000 .)

Table 5 (a) shows that, if one wants to be $95 \%$ confident that one system is better than another based on 20 topics, he should look for absolute differences of at least 0.11 in AveP and 0.10 in Q-measure, but that RR and O-measure cannot guarantee this confidence level. That is, the swap rates of $\mathrm{RR}$ and $\mathrm{O}$-measure do not fall below $5 \%$ even when the absolute differences are very large. Hence, metrics based on one relevant document are less sensitive than those based on all relevant documents, regardless of whether graded relevance is used or not.

Table 5 (b) shows that, using O-measure averaged across 20 topics, $90 \%$ confidence is guaranteed if the performance difference is at least 0.20 , or $0.20 / 0.8792=23 \%$ in terms of relative difference. $16.5 \%$ of all trial comparisons actually had this difference. Whereas, RR still cannot discriminate among systems with $90 \%$ confidence. This suggests that $O$-measure may be a little more sensitive than $R R$, due to the use of graded relevance.

Table 5 (c) shows that RR finally manages to discriminate among systems at $80 \%$ confidence. Note that O-measure still appears to be slightly more sensitive than RR, and that AveP and Q-measure are about twice as sensitive as $R R$ and $\mathrm{O}$-measure.

Table 6 shows a similar table based on the Japanese data. With this data set, all four metrics managed to guarantee $95 \%$ confidence. Again, it can be observed that AveP and $\mathrm{Q}$-measure are clearly more sensitive than $\mathrm{RR}$ and $\mathrm{O}$-measure. On the other hand, O-measure does not seem to be superior to RR in terms of sensitivity for this data set. That is, the use of graded relevance does not help much for the purpose of discriminating the Japanese document retrieval systems. This is probably because there are relatively few S-relevant documents in the NTCIR-3 CLIR Japanese test collection (See Section 4).

It can also be observed that, while the Chinese and the Japanese results are quite similar in terms of the required absolute/relative differences (Columns (i) and (iii)), they are 
quite different in terms of absolute sensitivity (Column (iv)). This implies that the 30 Japanese document runs are considerably easier to discriminate than the 30 Chinese ones. Recall that both the Buckley/Voorhees and Voorhees/Buckley methods depend not only on the test collection but also on the set of runs: It remains to be seen how well our findings apply to other experimental environments such as the TREC/NTCIR Web tracks.

\section{Conclusions}

This paper examined the problem of evaluating systems that aim at finding one highly relevant document with high precision. Using test collections from the NTCIR-3 CLIR track, we compared four IR metrics, based on binary/graded relevance and designed for finding one/all relevant document(s). One of them was $\mathrm{O}$-measure which we introduced in this paper. Our main findings are:

- In both binary and graded relevance environments, the task of finding one relevant document with high precision is not the same as that of finding all relevant documents with high precision. (That is, the best search strategies for Tasks C and D in Fig. 1 may be different from those for Tasks A and B.) The system rankings based on one relevant document is substantially different from, and less stable and sensitive than, those based on all relevant documents. Specifically, if one chooses to use RR or O-measure for IR evaluation instead of traditional metrics such as AveP, larger topic sets are probably required in order to obtain reliable results.

- The task of finding one highly relevant document with high precision is not the same as that of finding any one relevant document with high precision. (That is, the best search strategies for Task D may be different from those for Task C.) For the former task, O-measure with small gain values may be useful as it is highly correlated with $\mathrm{RR}$ and yet takes graded relevance into account. Moreover, O-measure is probably more stable than RR and at least as sensitive as RR.

It may be possible to devise a more stable metric for the task of finding one highly relevant document, which looks at how every relevant document is retrieved just like Q-measure but assigns a high gain value to the first retrieved relevant document only. However, such a metric would probably be less interpretable. Furthermore, as we discussed in Section 2.4, O-measure may not be suitable for an IR environment in which the user can easily identify a highly relevant document ranked below a partially relevant one in the presented ranked list. We shall discuss a different metric suitable for such situations elsewhere ${ }^{14)}$.

As the analyses reported in this paper depend on the NTCIR-3 CLIR data sets, it remains to be seen how well our findings apply to other experimental and practical search environments.

Finally, as mentioned earlier, we have already conducted experiments using the samplingwith-replacement version of the Voorhees/ Buckley method ${ }^{11)}$. We are currently exploring a direct application of the bootstrap ${ }^{4)}$ for comparing the stability and sensitivity of different IR metrics, where a bootstrap sample $Q_{i}^{*}$ is created from the original topic set $Q$ by sampling with replacement, and $\left|Q_{i}^{*}\right|=|Q|$. We plan to discuss this approach in our future work.

Acknowledgments We thank the IPSJ TOD Reviewers and the FIT 2005 Reviewers for their helpful comments.

\section{References}

1) Buckley, C. and Voorhees, E.M.: Evaluating Evaluation Measure Stability, ACM SIGIR 2000 Proceedings, pp.33-40 (2000).

2) Chen, K.-H., et al.: Overview of CLIR Task at the Third NTCIR Workshop, NTCIR-3 Proceedings (2003).

3) Cronen-Townsend, S., Zhou, Y. and Croft, W.B.: Predicting Query Performance, $A C M$ SIGIR 2002 Proceedings, pp.299-306 (2002).

4) Efron, B. and Tibshirani, R.J.: An Introduction to the Bootstrap, Chapman \& Hall/CRC (1993).

5) Eguchi, K., et al.: Overview of the Web Retrieval Task at the Third NTCIR Workshop, NTCIR-3 Proceedings (2003).

6) Järvelin, K. and Kekäläinen, J.: Cumulated Gain-Based Evaluation of IR Techniques, ACM Transactions on Information Systems, Vol.20, No.4, pp.422-446 (2002).

7) Kekäläinen, J.: Binary and Graded Relevance in IR evaluations - Comparison of the Effects on Ranking of IR Systems, Information Processing and Management, Vol.41, pp.1019-1033 (2005).

8) Sakai, T.: New Performance Metrics based on Multigrade Relevance: Their Application to Question Answering, NTCIR-4 Proceedings 
(2004).

9) Sakai, T.: A Note on the Reliability of Japanese Question Answering Evaluation, IPSJ SIG Technical Reports 2004-FI-77, pp.57-64 (2004).

10) Sakai, T.: Ranking the NTCIR Systems based on Multigrade Relevance, AIRS 2004 Revised Selected Papers, Lecture Notes in Computer Science 3411, pp.251-262, Springer (2004).

11) Sakai, T.: The Effect of Topic Sampling on Sensitivity Comparisons of Information Retrieval Metrics, NTCIR-5 Proceedings, pp.505-512 (2005).

12) Sakai, T.: An Evaluation Metric for the Task of Retrieving One Highly Relevant Document with High Precision (in Japanese), FIT 2005 Information Technology Letters LD-002, pp.69-72 (2005).

13) Sakai, T.: The Reliability of Metrics based on Graded Relevance, AIRS 2005 Proceedings, Lecture Notes in Computer Science 3689, pp.1-16 (2005).

14) Sakai, T.: A Further Note on Evaluation Metrics for the Task of Finding One Highly Relevant Document, IPSJ SIG Technical Reports 2006-FI-82, to appear (2006).

15) Sanderson, M. and Zobel, J.: Information Retrieval System Evaluation: Effort, Sensitivity, and Reliability, ACM SIGIR 2005 Proceedings, pp.162-169 (2005).

16) Shah, C. and Croft, W.B.: Evaluating High Accuracy Retrieval Techniques, ACM SIGIR 2004 Proceedings, pp.2-9 (2004).

17) Soboroff, I.: On Evaluating Web Search with Very Few Relevant Documents, ACM SIGIR 2004 Proceedings, pp.530-531 (2004).

18) Voorhees, E.M.: Evaluation by Highly Relevant Documents, ACM SIGIR 2001 Proceedings, pp.74-82 (2001).

19) Voorhees, E.M. and Buckley, C.: The Effect of Topic Set Size on Retrieval Experiment Error, ACM SIGIR 2004 Proceedings, pp.316-323 (2002).

\section{Appendix}

\section{A.1 Theorems}

\section{A.1.1 Optimality Theorems}

Definition 1 An ideal ranked output for a given topic with $R=\sum_{X} R(X)$ relevant documents is one s.t. isrel $(r)=1$ for $1 \leq r \leq R$ and $g(r) \leq g(r-1)$ for $r>1$. That is, the $R$ positive gain values that are available for the topic must be enumerated exhaustively in decreasing order from the top of the list. This implies that the system output size $L \geq R$.

The gain and the cumulative gain at Rank $r$ of an ideal ranked output are denoted by $g_{I}(r)$ and $c g_{I}(r)$ throughout this paper. It is clear that

$$
g_{I}(r)>0
$$

for $r \leq R$, and that

$$
g_{I}(r)=0
$$

for $r>R$. Thus,

$$
c g_{I}(r)=c g_{I}(R)=\sum_{X} R(X) \operatorname{gain}(X)
$$

holds for $r \geq R$. That is, $c g_{I}(r)$ is a constant for $r \geq R$. It is also clear that

$$
c g(r) \leq c g_{I}(r)
$$

holds for any system output and for any $r$.

Lemma 1 If $c g(r)=c g_{I}(r)$ and $r \leq R$, then $\operatorname{count}(r)=r$.

Proof: If $r=1$, then $c g(r)=c g_{I}(r)$ can be rewritten as $g(1)=g_{I}(1)$. Thus, from Eq. (11), $g(1)>0$. That is, the first document in the system output is relevant. Therefore, $\operatorname{count}(1)=1$ holds.

If $1<r \leq R$, let us assume that a document at Rank $j(\leq r)$ in the system output is nonrelevant, so that $g(j)=0$. In this case, $c g(r)=c g_{I}(r)$ can be rewritten as

$$
\sum_{1 \leq i \leq r, i \neq j} g(i)=\sum_{1 \leq i \leq r} g_{I}(i)=k
$$

where $k$ is a positive constant (Recall Eq. (11)). Now, Eq. (15) implies that there exists another ranked output s.t.

$$
c g(r-1)=\sum_{1 \leq i \leq r-1} g(i)=k .
$$

That is, it is possible to achieve a cumulative gain of $k$ at Rank $r-1$. However, from Eq. (15),

$$
\begin{aligned}
c g_{I}(r-1) & =\sum_{1 \leq i \leq r} g_{I}(i)-g_{I}(r) \\
& =k-g_{I}(r)<k .
\end{aligned}
$$

Thus, from Eqs. (16) and (18),

$$
c g(r-1)>c g_{I}(r-1)
$$

which contradicts with Eq. (14). Therefore, documents from Ranks 1 through $r$ must all be relevant. Thus count $(r)=r$ holds.

Lemma 2 If $r>R$, then $B R(r)<1$.

Proof: By definition,

$$
\operatorname{count}(r) \leq R
$$

holds for any $r$. Given that $r>R$, using Eqs. (14) and (20),

$$
\begin{aligned}
B R(r) & =\frac{c g(r)+\operatorname{count}(r)}{c g_{I}(r)+r} \\
& \leq \frac{c g(r)+R}{c g_{I}(r)+r}<1 .
\end{aligned}
$$


Lemma $3 \quad B R(r)=1$ if and only if $c g(r)=$ $c g_{I}(r)$ and $r \leq R$.

Proof: Given $B R(r)=1$, this can be rewritten as

$$
c g(r)+\operatorname{count}(r)=c g_{I}(r)+r .
$$

But since both Eq. (14) and

$$
\operatorname{count}(r) \leq r
$$

hold in general, $c g(r)=c g_{I}(r)$ is a necessary condition for satisfying Eq. (23). Moreover, from Lemma 2, $B R(r)=1$ implies that $r \leq R$.

Conversely, given that $c g(r)=c g_{I}(r)$ and $r \leq R$ hold, count $(r)=r$ holds from Lemma 1 . Therefore, Eq. (23) holds. That is, $B R(r)=1$.

Lemma 4 If $g(r)=c g_{I}(r)$ and $r \leq R$, then $r=1$.

Proof: If $S$ denotes the highest relevance level, then $g_{I}(1)=\operatorname{gain}(S)$. Now, let us assume that $r>1$. Then, $g(r)=c g_{I}(r)$ can be rewritten as

$$
g(r)=\operatorname{gain}(S)+\sum_{1<i \leq r} g_{I}(i)
$$

But since $r \leq R$, it follows from Eqs. (11) and (25) that

$$
g(r)>\operatorname{gain}(S) \text {. }
$$

This is a contradiction, since gain $(S)$ is the highest gain value that can be achieved. Therefore $r=1$.

Theorem 1 -measure $=1$ if and only if the system output has any one of the most relevant documents at Rank 1.

Proof: Given that $O$-measure $=1$, the system output contains at least one relevant document, so let $r^{\prime}$ be the rank of the first relevant one in the output. Thus $B R\left(r^{\prime}\right)=1$, and therefore and

$$
r^{\prime} \leq R
$$

$$
c g\left(r^{\prime}\right)=c g_{I}\left(r^{\prime}\right)
$$

hold from Lemma 3 . Hence, by definition of $r^{\prime}$,

$$
g\left(r^{\prime}\right)=c g_{I}\left(r^{\prime}\right)
$$

holds. Using Eqs. (27) and (29) with Lemma 4, we obtain $r^{\prime}=1$. Moreover, substituting this into $O$-measure $=1$ yields

Thus

$$
g(1)+1=c g_{I}(1)+1=g_{I}(1)+1
$$

$$
g(1)=g_{I}(1)=\operatorname{gain}(S) .
$$

Therefore, the system output has one of the most relevant documents at Rank 1 .

Conversely, given that the system output has any one of the most relevant documents at Rank 1, then $r^{\prime}=1$ and Eq. (31) hold. Therefore,

$$
O \text {-measure }=\frac{g(1)+1}{g_{I}(1)+1}=1 .
$$

Theorem 2 Q-measure $=1$ if and only if the system output is an ideal one.

Proof: Given that $Q$-measure $=1$, this can be rewritten as

$$
\begin{aligned}
R & =\sum_{1 \leq r \leq L} i \operatorname{srel}(r) B R(r) \\
& \leq \sum_{1 \leq r \leq L} i \operatorname{srel}(r)
\end{aligned}
$$

since $B R(r) \leq 1$. But in general,

$$
R \geq \sum_{1 \leq r \leq L} \operatorname{isrel}(r)
$$

holds by definition and therefore

$$
\begin{aligned}
R & =\sum_{1 \leq r \leq L} i \operatorname{srel}(r) B R(r) \\
& =\sum_{1 \leq r \leq L} i \operatorname{srel}(r)
\end{aligned}
$$

must hold. Firstly, the above implies that the system output includes all relevant documents. Secondly, it implies that $B R(r)=1$ for all $r$ s.t. isrel $(r)=1$. Taken together, the system output must satisfy $B R(r)=1$ for all relevant documents, where $r$ is the rank of each relevant document retrieved. Thus, from Lemma 3, the system output must satisfy $c g(r)=c g_{I}(r)$ and $r \leq R$ for all relevant documents. In other words, $c g(r)=c g_{I}(r)$ holds for any $r(\leq R)$. Therefore the system output must be an ideal one.

Conversely, given that the system output is an ideal one, then only the top $R$ documents are relevant, and $c g(r)=c g_{I}(r)$ holds for each $r(\leq R)$. Therefore, from Lemma $3, B R(r)=1$ for each $r(\leq R)$. Thus,

$$
\begin{aligned}
Q \text {-measure } & =\frac{1}{R} \sum_{1 \leq r \leq R} B R(r) \\
& =\frac{1}{R} \sum_{1 \leq r \leq R}=\frac{1}{R} * R=1 .
\end{aligned}
$$

\section{A.1.2 Binary Relevance Theorems}

In a binary relevance environment, we can let each retrieved relevant document yield a gain value of one. Thus,

$$
c g(r)=\operatorname{count}(r)
$$

holds for $r \geq 1$,

$$
c g_{I}(r)=r
$$

holds for $r \leq R$, while

$$
c g_{I}(r)=R
$$

holds for $r>R$.

Lemma 5 In a binary relevance environment, $B R(r)=P(r)$ holds if and only if $r \leq R$. 
Proof: Given that $B R(r)=P(r)$ holds, let us assume that $r>R$. Under this assumption, we obtain the following from Eqs. (40) and (42):

$$
B R(r)=\frac{2 \operatorname{count}(r)}{R+r} .
$$

Since this must equal $P(r)$,

$$
\frac{2 \operatorname{count}(r)}{R+r}=\frac{\operatorname{count}(r)}{r}
$$

holds. Thus, $2 r=R+r$ and hence $r=R$, which contradicts with our assumption. That is, $r \leq R$ must hold.

Conversely, given that $r \leq R$ in a binary relevance environment, we obtain the following from Eqs. (40) and (41):

$$
B R(r)=\frac{2 \operatorname{count}(r)}{2 r}=P(r) .
$$

Lemma 6 In a binary relevance environment, $B R(r)>P(r)$ holds if and only if $r>R$.

Proof: From Lemma 5, we only need to prove that $B R(r)>P(r)$ holds if $r>R$.

Given that $r>R$, from Eq. (43) we obtain

$$
B R(r)>\frac{2 \operatorname{count}(r)}{2 r}=P(r) \text {. }
$$

Theorem 3 If a system output contains its first relevant document at Rank $r^{\prime}$ in a binary relevance environment, $O$-measure $=R R$ holds if and only if $r^{\prime} \leq R$.

Proof: This is a corollary from Lemma 5.

Theorem 4 If a system output contains its first relevant document at Rank $r^{\prime}$ in a binary relevance environment, $O$-measure $>R R$ holds if and only if $r^{\prime}>R$.

Proof: This is a corollary from Lemma 6 .
Theorem 5 In a binary relevance environment, $Q$-measure $=$ AveP holds if and only if there is no relevant document below Rank $R$.

Proof: This is a corollary from Lemmas 5 and 6 .

Theorem 6 In a binary relevance environment, Q-measure $>$ AveP holds if and only if there is at least one relevant document below Rank $R$.

Proof: This is a corollary from Lemmas 5 and 6 .

(Received September 12, 2005)

(Accepted November 5, 2005)

(Released March 29, 2006)

\section{(Editor in Charge: Manabu Ohta)}

(Paper version of this article can be found in the IPSJ Transactions on Databases, Vol.47 No.SIG4(TOD29), pp.13-27.)

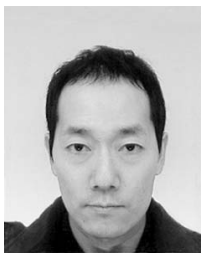

Tetsuya Sakai was born in 1968. He received a Master's degree in Engineering from Waseda University in 1993 and joined Toshiba Corporate R\&D Center in the same year. $\mathrm{He}$ received a Ph.D. from Waseda University in 2000 for his work on information retrieval and filtering systems. From 2000 to 2001, he was a visiting researcher at University of Cambridge Computer Laboratory. He is currently a Research Scientist at Toshiba Corporate R\&D Center Knowledge Media Laboratory. He is a member of IPSJ and IEICE. 